\title{
A Whole-Course and Multidisciplinary Nutrition Management Model for Breast Cancer: A Typical Case Report
}

\author{
Yuanzhen Luo ${ }^{1,2,3 *}$, Li Shi1,2,3*, Linfei Liu ${ }^{1,2,3}$, Rong Chen ${ }^{1,2,3}$, Huiting Zhang1,2,3\# \\ ${ }^{1}$ State Key Laboratory of Oncology in South China, Guangzhou, China \\ ${ }^{2}$ Collaborative Innovation Center for Cancer Medicine, Guangzhou, China \\ ${ }^{3}$ Department of Breast Cancer, Sun Yat-sen University Cancer Center, Guangzhou, China \\ Email: "zhanght@sysucc.org.cn
}

How to cite this paper: Luo, Y.Z., Shi, L., Liu, L.F., Chen, R. and Zhang, H.T. (2021) A Whole-Course and Multidisciplinary $\mathrm{Nu}$ trition Management Model for Breast Cancer: A Typical Case Report. Advances in Breast Cancer Research, 10, 173-183.

https://doi.org/10.4236/abcr.2021.104015

Received: August 18, 2021

Accepted: September 12, 2021

Published: September 15, 2021

Copyright (c) 2021 by author(s) and Scientific Research Publishing Inc. This work is licensed under the Creative Commons Attribution International License (CC BY 4.0).

http://creativecommons.org/licenses/by/4.0/

\section{Open Access}

\begin{abstract}
Breast cancer patients face different nutritional problems at each stage. However, the nutrition of breast cancer patients has not been taken seriously. As a result, some patients cannot tolerate treatment due to poor nutrition, thus affecting the prognosis. This study aims to introduce a typical case to explore the whole-course (started at admission and ended 5 years after surgery) and Multidisciplinary Teams (MDTs) (comprising physician, primary nurse, case manager, nutrition liaison and nutrition specialist nurse) nutritional management mode of breast cancer patients. The patient successfully completed the scheduled treatment by implementing the whole-course and multidisciplinary nutrition management. The mode can prospectively and dynamically estimate the changes in patients' nutritional status, and provide timely nutritional intervention to promote patient outcomes.
\end{abstract}

\section{Keywords}

Breast Cancer, Nutrition, Whole-Course, Multidisciplinary, Case Management

\section{Introduction}

A number of international studies have indicated that the incidence of malnutrition in patients with malignant tumours is higher than one with other diseases. One of these studies has shown that $57.88 \%$ of patients with malignant tumours are complicated by various levels of malnutrition. The incidence of malnutrition in patients with breast tumour is $20.5 \%$ [1] [2] [3]. Both breast cancer itself, as a *Yuanzhen Luo, Li Shi should be considered joint first author.

"Huiting Zhang should be considered corresponding author. 
malignant tumour, and complicated and long-lasting treatment, which covers surgery, chemotherapy, radiotherapy, targeted therapy and endocrine therapy, make breast cancer patients face various nutrition problems at different stages [4] [5] [6] [7]. For instance, during the diagnosis period, patients may consume a lot of energy and protein due to the rapid growth of the malignant tumour, resulting in dyscrasia. On the other hand, patients had reduced intake due to lack of knowledge and negative emotions such as anxiety and fear [8] [9] [10]. Moreover, patients may experience hyperglycaemia, abnormal protein catabolism and negative nitrogen balance during the perioperative period due to systemic stress response caused by preoperative fasting and surgical trauma. In addition, during chemoradiotherapy, patients frequently experience gastrointestinal reactions such as nausea, vomiting, oral ulcer, abnormal liver and kidney function and immune injury due to chemotherapy drugs and radiotherapy reactions [11] [12] [13]. On the other hand, during endocrine therapy, patients often experience dyslipidaemia due to drug reactions, and their appetite can be affected by perimenopausal syndrome reactions (hectic fever and irritability) and other discomforts. Malnutrition may lead to a decrease in the tolerance and sensitivity of patients to tumour treatment, resulting in poor clinical outcomes, such as complications and increased risk of mortality, prolonged hospital stay, increased frequency of rehospitalisation and increased medical expenses [14]. Therefore, nutrition management for patients is of vital importance. However, nutrition problems in patients with malignant tumours of the digestive system have received most of the attention at present [15] [16], while those in patients with breast cancer are not taken seriously. As a result, some breast cancer patients cannot tolerate treatment due to poor nutrition, thus affecting the prognosis. Moreover, the attention to the nutrition problems in patients with malignant tumours of the digestive system focuses on a certain period of treatment, nevertheless, there are few studies on long-term whole-course nutrition management for patients.

Therefore, we intend to explore a whole-course nutrition management model for breast cancer patients by introducing a representative successful case of the wholecourse nutrition management for a patient with breast cancer in our hospital.

\section{Methods}

\subsection{Case Presentation}

A 26-year-old female with right breast mass has been performed breast conserving surgery with axillary lymph node dissection on February 20, 2017. The postoperative pathologic results revealed invasive ductal carcinoma, and axillary lymph nodes showed five sites of metastatic carcinoma, ER (30\%+), PR (-), HER-2 (3+) and Ki-67 $(40 \%+)$. The combination of TE*4 (epirubicin $130 \mathrm{mg}+$ paclitaxel liposome $240 \mathrm{mg}$ ) + $\mathrm{TP}^{\star} 4$ (paclitaxel liposome $240 \mathrm{mg}+$ carboplatin $600 \mathrm{mg}$ ) adjuvant chemotherapy and trastuzumab (the dose was adjusted with the weight; the first dose was $8 \mathrm{mg} / \mathrm{Kg}$ and the subsequent dose $6 \mathrm{mg} / \mathrm{Kg}$ ) targeted therapy began in March 2017. In addition, radiotherapy was performed from August to 
October 2017. Since October 2017, the patient has been receiving endocrine therapy with subcutaneous injection of leuprorelin $3.75 \mathrm{mg} \mathrm{Q} 4 \mathrm{~W}+$ oral administration of tamoxifen $10 \mathrm{mg}$. This is a common treatment for breast cancer. This is a typical case of breast cancer underwent five conventional breast cancer therapies.

\subsection{Case Analysis}

Characteristics of cases (Table 1 and Table 2):

This is a common treatment for breast cancer. This is a typical case of breast cancer underwent five conventional breast cancer therapies. These treatments can cause patient to experience many side effects that affect her nutrition.

The patient's weight decreased from $55 \mathrm{~kg}$ to $52 \mathrm{~kg}$ within 1 month prior to surgery.

The patient lacks proper knowledge of diet.

\section{Measurements}

The patient-generated subjective global assessment (PG-SGA) scale was used to

Table 1. Sociodemographic and clinical characteristics of the patient.

\begin{tabular}{cc}
\hline Project & Characteristics \\
\hline Age & 26 \\
Gender & Female \\
Professional & Office clerk \\
Marital status & Unmarried \\
Nutritional assessment & Within 1 month after the onset, the patient's weight \\
decreased from 55 kg to 52 kg
\end{tabular}

Table 2. The treatment process.

\begin{tabular}{|c|c|c|}
\hline Time & Treatment of type & Treatment options \\
\hline $2017-2-20$ & Surgery & $\begin{array}{l}\text { Breast conserving surgery withaxillary } \\
\text { lymph node dissection }\end{array}$ \\
\hline 2017-3-7 2017-8-9 & Chemotherapy & $\begin{array}{c}\mathrm{TE}^{*} 4 \text { (epirubicin } 130 \mathrm{mg}+\text { paclitaxel liposome } \\
240 \mathrm{mg})+\mathrm{TP}{ }^{*} 4 \text { (paclitaxel liposome } 240 \mathrm{mg} \\
+ \text { carboplatin } 600 \mathrm{mg})\end{array}$ \\
\hline $2017-3-7 \sim 2017-8-9$ & Targeted therapy & Trastuzumab targeted therapy \\
\hline $2017-8-23 \sim 2017-10-3$ & Radiation therapy & Radiation area: Right armpit \\
\hline $\begin{array}{l}2017-10-4 \sim \\
\text { up to now }\end{array}$ & Endocrine therapy & $\begin{array}{l}\text { Injection of leuprorelin } 3.75 \mathrm{mg} \mathrm{Q} 4 \mathrm{~W} \\
+ \text { oral administration of tamoxifen } 10 \mathrm{mg}\end{array}$ \\
\hline
\end{tabular}


determine the nutritional risk of the patient during the treatment [17]. This scale is a nutritional status assessment method explicitly designed for cancer patients [18] and is composed of two parts: self-assessment by the patient and assessment by medical staff. The former involves weight change, food consumption, symptoms and activity and body functions; and the total scores of the four aspects form Score A. The latter includes the relationship between disease and nutritional needs (Score B), metabolic needs (Score C) and physical examination (Score D). According to the sum of the four parts (Score A D), $0 \sim 1$ point means good nutrition, $2 \sim 8$ points mean suspected or moderate malnutrition and 9 points mean severe malnutrition. The time points for PG-SGA are on admission; on the $1^{\text {st }}$ day after surgery; before chemotherapy; at early, middle and late stages of chemotherapy; before radiotherapy; before endocrine therapy; in the $3^{\text {rd }}, 6^{\text {th }}$ and $12^{\text {th }}$ month of endocrine therapy and once a year afterwards, until the end of endocrine therapy. If the score is higher than 9 points, the nutritional status should be reassessed within 1 week.

\section{Intervention Programme}

The patient-centred Multidisciplinary Teams (MDTs) of 'case manager (family-based follow-up)/primary nurse (during hospitalisation), nutrition liaison, nutrition nurse and physician' were established for the whole-course nutrition management for the patient, including the preoperative, postoperative, chemoradiotherapy and endocrine therapy periods. The main model of operation is as follows: 1) Nutrition nurse: senior nurses will be chosen and sent by the hospital to attend a 3-month provincial nutrition nurse training course and to obtain the nutritionist qualification certificate. These nurses compete for full-time positions of nutrition nurse upon their return to the hospital. Nutrition nurses and nutritionists form a hospital nutrition group to be responsible for professional training on nutrition knowledge for nutrition liaison, regular nutrition nursing rounds in each department and instruction of nutrition problems encountered in the work of various departments; 2) Nutrition liaison: each inpatient ward should arrange a nutrition liaison as a bridge between clinical departments and the nutrition nurse. The nutrition liaison should take part in nutrition courses conducted by the nutrition team every month and instruct nurses in the department to administer nutrition assessment and interventions for patients about what has been learned. Any nutrition problem of patients that cannot be addressed should be reported to the nutrition team without delay for instruction from nutrition nurse; 3) Primary nurse or case manager: during the treatment, the nutritional status of patients is monitored and assessed by the primary nurse (during hospitalisation)/case manager (family-based follow-up); 4) Physicians: include the supervising physician and the nutritionist; when patients' nutrition problems are evaluated as severe or may affect the treatment progress, the physician should be provided with feedback for joint intervention and may adjust the dosage or prescribe nutrition prescriptions. 


\subsection{Preoperative Nutrition Problems and Interventions}

Within 1 month after the onset, the patient's weight decreased from $55 \mathrm{~kg}$ to 52 $\mathrm{kg}$. The patient's total score of the PS-SGA scale was 9 points. Hence, she was assessed with severe malnutrition. The primary nurse immediately referred to the physician, nutrition liaison and case manager and consulted a nutrition nurse. After the diagnosis, the patient was found to have lost appetite due to worry and anxiety and, due to the wrong cognition that protein-rich foods (such as eggs and milk) would increase the risk of disease progression. The results of the preoperative biochemical analysis showed that albumin (ALB) was as low as 38.7 $\mathrm{g} / \mathrm{L}$. The nutrition nurse advised the patient to had three meals on time and had $250 \mathrm{~g} \sim 300 \mathrm{~g}$ staple food, $150 \mathrm{~g}$ meat and corresponding fat and oil, one egg, milk and fruit, producing $1200 \sim 1500 \mathrm{kcal}$ of energy. And the primary nurse politely addressed the patient's and her family's questions to relieve the patient's worries and change the original wrong diet concept. After the interventions, the patient's food consumption returned to $90 \%$ of her normal intake. Additionally, her weight was maintained at $52 \mathrm{~kg}$. The patient was reassessed with the PG-SGA scale with 5 points before surgery.

\subsection{Postoperative Nutrition Problems and Interventions}

The patient experienced vomiting, wound pain and arm soreness on the affected side on the first day after surgery. The score of the PG-SGA scale was 10 points. After reporting to the physician, the primary nurse disposed appropriately as follows: used antiemetic and analgesic drugs, touch the affected side arm with manual lymphatic drainage. The patient's postoperative diet after the relief of such discomforts as vomiting and pain was instructed: on the first day after surgery, breakfast should be rice porridge and other liquid food; lunch and dinner should be normal food. The patient might have more meals a day but less food for each. Vegetables, fruits, eggs, milk and meat should be taken. During the wound healing period, three meals should be kept normal, and the intake should be the same as before surgery to ensure adequate protein intake.

Upon discharge, the primary nurse and case manager handed over the patient's nutritional status. The patient had a good appetite; her weight increased by $1 \mathrm{~kg}$, PG-SGA score was 4 points and the wound healed well, but she did not want to get up and move most of the time due to the discomfort of the wound and the arm on the affected side; the time in bed or in a chair was not longer than half a day. As a result, the case manager carried out health education on the patient and her family and instructed the patient's diet and activities at home.

\subsection{Nutrition Problems and Interventions during Chemoradiotherapy and Targeted Therapy}

On the day of chemotherapy, the primary nurse introduced side effects of chemotherapy and the corresponding countermeasures to minimise the risk of adverse reactions of chemotherapy drugs and to reduce the patient's psychological 
burden worrying about nausea and vomiting. The primary nurse also advised the patient some tips on diet to help her cope successfully with the gastrointestinal reactions during chemotherapy. The case manager should follow-up patients by phone $7 \sim 10$ days after each course of chemotherapy. The physician and nutrition liaison should be immediately informed of the patients' problems in order to provide professional support. During the follow-up, the case manager discovered that the patient had the following problems during chemotherapy. During the $1^{\text {st }}$ chemotherapy course, the patient had Grade I constipation and was instructed to exercise moderately, the case manager increase the intake of water and vegetables and fruits and use glycerine enema. During the $4^{\text {th }}$ chemotherapy course, the patient had Grade II oral mucositis and plaque ulcer in the oral cavity, affecting food intake, the case manager urge the patient to brush her teeth with a soft brush after meals, and contacted the physician to prescribe compound Vitamin B12 solution for exterior coating and encouraged the patient to take vitamin-rich, protein-rich, digestible semi-liquid food and supplements with little residue. After the $5^{\text {th }}$ and $6^{\text {th }}$ chemotherapy courses, the patient had Grade II nausea and loss of appetite, the case manager advised the patient to drink as little water as possible before and after meals and not to lie down immediately after meals in order to avoid food reflux and nausea. After the $7^{\text {th }}$ chemotherapy course, the patient had Grade I myelosuppression and slightly decreased White Blood Cells (WBCs) and was instructed to pay attention to personal hygiene and keep track of the blood count. During the chemotherapy, the patient's PG-SGA score was $3 \sim 6$ points.

During the targeted therapy, the patient was instructed to monitor the cardiac function regularly and the dietary nutritional status was monitored. No nutrition-related complications occurred during this period.

The patient was instructed to use compound Vitamin B12 solution to avoid radioactive oral mucositis during radiotherapy. At the follow-up, the case manager learned that the patient had mild xerostomia, but had good appetite and improved intake. The patient's weight increased to $57 \mathrm{~kg}$. The PG-SGA score after the radiotherapy was 2 points.

\subsection{Nutrition Problems and Interventions during Endocrine Therapy}

The timeframes for follow-up by the case manager are before the endocrine therapy; the $3^{\text {rd }}, 6^{\text {th }}$ and $12^{\text {th }}$ month after the beginning of endocrine therapy and once a year, until the end of the endocrine therapy within 5 years. Each follow-up is based on the patient's blood lipid, calcium, weight and PG-SGA score. The case manager should provide the physician and the nutrition liaison with feedback on these indicators.

Before the endocrine therapy, the physician and case manager instructed the patient to adjust the original dietary structure: increasing the consumption of vegetables, fruits and grains, minimising the intake of fat and sugar and supple- 
menting vitamins and fibre to reduce fat absorption. In addition to the diet, the patient should also be advised to take proper exercise and regular rest and to undergo regular re-examination of uterine B-ultrasound and regularly track the changes in blood lipid and blood calcium. Elevated blood lipid was identified by biochemical re-examination in the patient when she had been taking tamoxifen for 3 months. The patient provided feedback to the case manager via WeChat. Since the patient was far away from our hospital, the case manager recommended local medical treatment and treatment with lovastatin ( $20 \mathrm{mg}$ orally/day) after consultation with the supervising physician and again emphasised the importance of low-sugar and low-fat diet. After a combination of diet and drug therapy, the re-examination in the 6 months of endocrine therapy revealed that the patient's blood lipid returned normal. At present, the patient is in the third year of endocrine therapy. So far, her blood lipid and blood calcium are within the normal range. During the endocrine therapy, the weight is kept at $57 \sim 58.5$ $\mathrm{kg}$. The PG-SGA score is always 1 point, requiring no intervention. During the treatment, routine follow-up and evaluation were maintained.

\section{Results}

The patient was followed for up to four years.The patient's laboratory indicators, weight and other indicators did not show any obvious abnormalities at different stages (Figure 1 and Figure 2) through the multidisciplinary whole-course nutrition management for the patient. And the abnormalities were treated promptly in combination with the results of PG-SAG (Table 3). Surgery, chemoradiotherapy and targeted therapy for the patient were successfully completed. Progress in

Laboratory Indicators
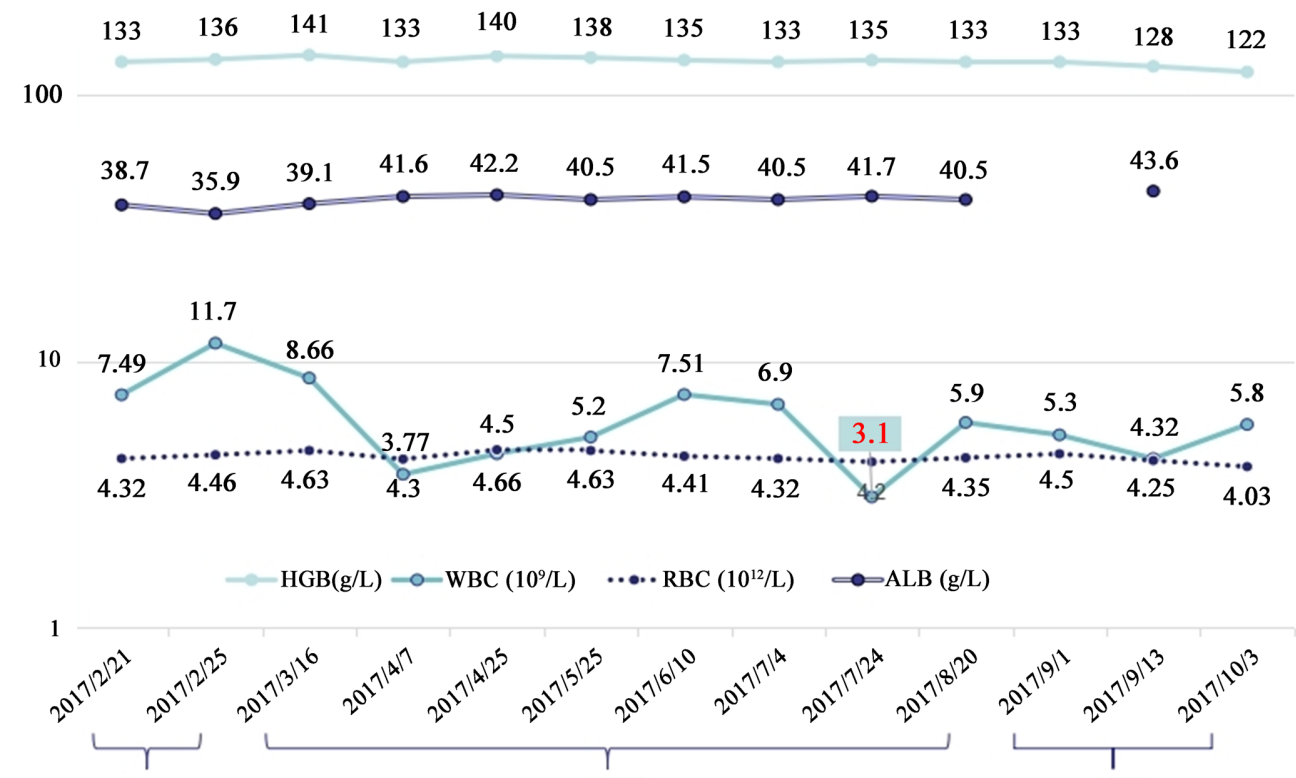

Perioperative period

Chemotherapy period

Radiotherapy period

Figure 1. Changes in the patient's laboratory indicators. Notes: HGB: haemoglobin; RBC: red blood cell; WBC: white blood cell; ALB: albumin. 


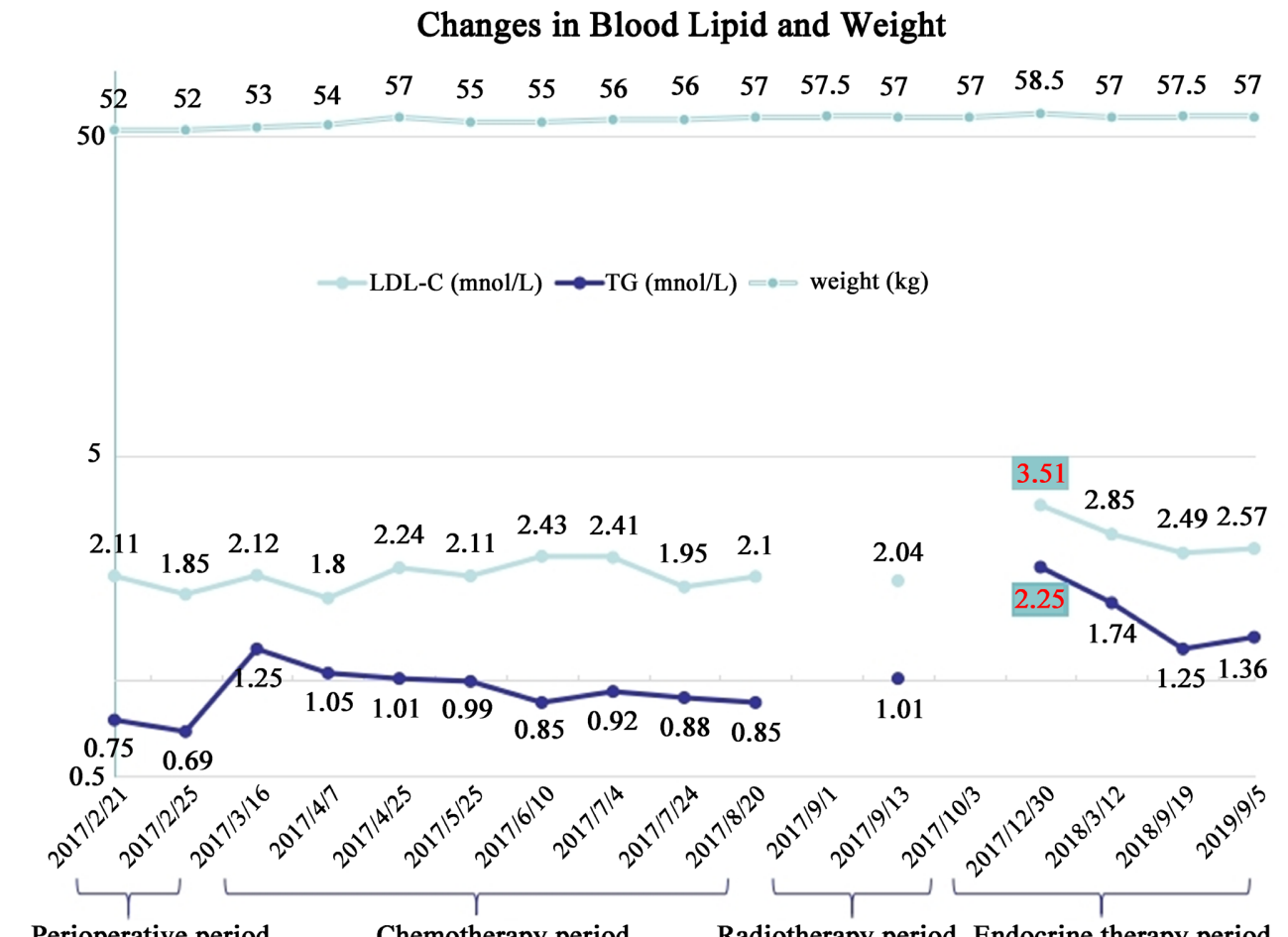

Perioperative period

Chemotherapy period

Radiotherapy period Endocrine therapy period

Figure 2. Changes in the patient's blood lipid and weight. Notes: LDL-C: low density lipoprotein cholesterol; TG: triglyceride.

Table 3. PG-SGA nutritional risk score of the patient.

\begin{tabular}{|c|c|c|c|c|c|c|c|c|c|c|c|c|}
\hline 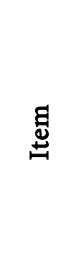 & 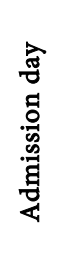 & 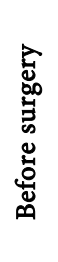 & 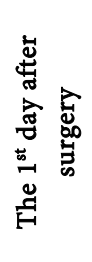 & 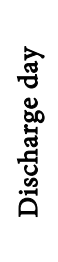 & 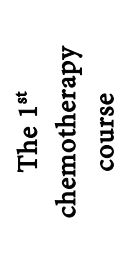 & 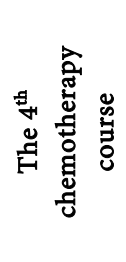 & 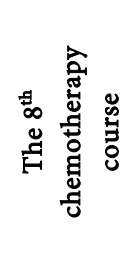 & 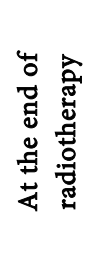 & 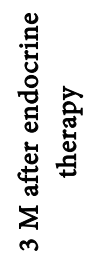 & 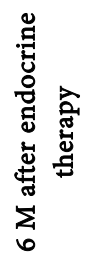 & 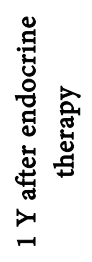 & 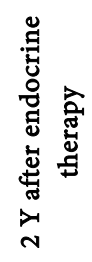 \\
\hline $\mathrm{A} 1$ & 4 & 3 & 2 & 1 & 1 & 2 & 0 & 0 & 0 & 0 & 0 & 0 \\
\hline $\mathrm{A} 2$ & 1 & 1 & 1 & 0 & 1 & 1 & 1 & 0 & 0 & 0 & 0 & 0 \\
\hline $\mathrm{A} 3$ & 3 & 0 & 3 & 0 & 1 & 2 & 1 & 1 & 0 & 0 & 0 & 0 \\
\hline $\mathrm{A} 4$ & 0 & 0 & 2 & 1 & 1 & 0 & 0 & 0 & 0 & 0 & 0 & 0 \\
\hline B & 1 & 1 & 2 & 2 & 1 & 1 & 1 & 1 & 1 & 1 & 1 & 1 \\
\hline $\mathrm{C}$ & 0 & 0 & 0 & 0 & 0 & 0 & 0 & 0 & 0 & 0 & 0 & 0 \\
\hline $\mathrm{D}$ & 0 & 0 & 0 & 0 & 0 & 0 & 0 & 0 & 0 & 0 & 0 & 0 \\
\hline Total & 9 & 5 & 10 & 4 & 5 & 6 & 3 & 2 & 1 & 1 & 1 & 1 \\
\hline
\end{tabular}

Notes: A1 means weight change; A2 means intake; A3 means symptoms; A4 means activities and physical functions; B means the relation between the disease and nutritional needs; $\mathrm{C}$ means metabolic needs; $\mathrm{D}$ means physical examination; Score $\mathrm{A}=\mathrm{A} 1+\mathrm{A} 2+\mathrm{A} 3+\mathrm{A} 4$; Total $=\mathrm{A}+\mathrm{B}+\mathrm{C}+\mathrm{D} ; \mathrm{M}$ means month; Y means year.

treatment was not affected by nutrition-related problems. The patient is now in the third year of endocrine therapy. The medication and nutrition conditions are still regularly followed up. 


\section{Discussion}

Our study reports a representative successful case of the whole-course nutrition management for a patient with breast cancer. To our knowledge, our study is the first to demonstrate the long-term effects of a management model of "wholecourse" and "multidisciplinary cooperation" on nutrition for patients with breast cancer. This study also illustrates a concrete model in China, which may help other researchers in implementing similar approaches or study designs.

A nutrition liaison was assigned in this model. The assessment and nursing intervention of the patient's nutritional status provided by the primary nurse (during hospitalisation)/case manager (family-based follow-up) were based on the instructions of the nutrition liaison. The assignment of the nutrition liaison reduced the work pressure of the nutrition nurse and improved the nutritional level of clinical nurses. In addition, this model took advantage of case management, in which the case manager cooperated with MDTs to provide the patient with whole-course tracking management during diagnosis, treatment and convalescence and played an important role in the whole-course management of the patient due to the lack of follow-up after discharge.

\section{Conclusion}

The whole-course nutrition is of great significant for patients with breast cancer. We carried out the whole-course management of the nutrition of patients with breast cancer by means of the multidisciplinary medical care cooperation model consisting of the primary nurse (during hospitalisation)/case manager (family-based follow-up), nutrition liaison, nutrition nurse and physician. This model is worthy of implementation and popularisation in nutrition management for patients.

\section{Ethical Approval}

The procedures used in this study adhered to the tenets of the Declaration of Helsinki.

\section{Acknowledgements}

The authors thank all the participants for their contributions to this study.

\section{Sources of Funding}

This work was supported by the Medical Research Fund of Guangdong Province (grant numbers: 2016A2020088).

\section{Conflicts of Interest}

The authors declare no conflicts of interest regarding the publication of this paper.

\section{References}

[1] Bossola, M. (2015) Nutritional Interventions in Head and Neck Cancer Patients 
Undergoing Chemoradiotherapy: A Narrative Review. Nutrients, 7, 265-276. https://doi.org/10.3390/nu7010265

[2] Steenhagen, E., van Vulpen, J.K., van Hillegersberg, R., May, A.M. and Siersema, P.D. (2017) Nutrition in Peri-Operative Esophageal Cancer Management. Expert Review of Gastroenterology \& Hepatology, 11, 663-672. https://doi.org/10.1080/17474124.2017.1325320

[3] Campillo, M.C.M., Fernández, J.M., Amo, S.M. and Rituerto, D.C. (2017) A Randomized Controlled Trial of Preoperative Oral Immuno Nutrition in Patients Undergoing Surgery for Colorectal Cancer: Hospital Stay and Health Care Costs. Cirugía y Cirujanos, 85, 393-400. https://doi.org/10.1016/j.circen.2017.11.008

[4] Gupta, D., Vaship, G., Lammersfeld, C.A. and Braun, D.P. (2011) Role of Nutritional Status in Predicting the Length of Stay in Cancer: A Systematic Review of the Epidemiological Literature. Annals of Nutrition and Metabolism, 5, 96-106. https://doi.org/10.1159/000332914

[5] Jeffe, D.B., Pérez, M., Cole, E.F., Liu, Y. and Schootman, M. (2016) The Effects of Surgery Type and Chemotherapy on Early-Stage Breast cancer Patients' Quality of Life over 2-Year Follow-Up. Annals of Surgical Oncology, 23, 735-743. https://doi.org/10.1245/s10434-015-4926-0

[6] Da, S.E., Carioca, A.A., Verde, S.M., da Conceição Quintaneiro, E. and Damasceno, N.R.T. (2015) Effect of Chemotherapy on Dietary Glycemic Index and Load in Patients with Breast Cancer and Their Relationships to Body Fat and Phase Angle. Nutrition and Cancer, 67, 587-593. https://doi.org/10.1080/01635581.2015.1019638

[7] Goss, A.M., Goree, L.L., Ellis, A.C., Chandler-Laney, P.C., Casazza, K., Lockhart, M.E., et al. (2013) Effects of Diet Macronutrient Composition on Body Composition and Fat Distribution during Weight Maintenance and Weight Loss. Obesity, 21, 1139-1142. https://doi.org/10.1002/oby.20191

[8] Zamanian, H., Amini-Tehrani, M., Jalali, Z., Daryaafzoon, M., Ala, S., Tabrizian, S., et al. (2021) Perceived Social Support, Coping Strategies, Anxiety and Depression among Women with Breast Cancer: Evaluation of a Mediation Model. European Journal of Oncology Nursing, 50, Article ID: 101892.

https://doi.org/10.1016/j.ejon.2020.101892

[9] Martín, B.R., Rodríguez, E.J.F., Galve, M.I.R. and Hernández, J.J.C. (2020) Study of Chemotherapy-Induced Cognitive Impairment in Women with Breast Cancer. International Journal of Environmental Research and Public Health, 17, Article No. 8896. https://doi.org/10.3390/ijerph17238896

[10] Ruby, N.S., Vijayakumar, C., Sundaramurthi, S., Sureshkumar, S., Kumbhar, U. and Balasubramanian, G. (2020) Knowledge and Attitude of Newly Diagnosed Breast Cancer Patients and Their Accompanying Attendants about Multimodality Treatment for Breast Cancer. Cureus, 12, Article No. e7915.

https://doi.org/10.7759/cureus.7915

[11] Spinelli, S., Mini, E., Monteleone, E., Angiolini, C. and Roviello, G. (2021) ALTERTASTE: Improving Food Pleasure and Intake of Oncology Patients Receiving Chemotherapy. Future Oncology, 17, 2573-2579. https://doi.org/10.2217/fon-2020-0871

[12] Prieto-Callejero, B., Rivera, F., Fagundo-Rivera, J., Romero, A., Romero-Martín, M., Gómez-Salgado, J., et al. (2020) Relationship between Chemotherapy-Induced Adverse Reactions and Health-Related Quality of Life in Patients with Breast Cancer. Medicine, 99, Article No. e21695. https://doi.org/10.1097/MD.0000000000021695

[13] Tang, N.L.S., Liao, C.D., Wang, X., Mo, F.K.F., Chan, V.T.C., Ng, R., et al. (2013) Role of Pharmacogenetics on Adjuvant Chemotherapy-Induced Neutropenia in Chinese Breast Cancer Patients. Journal of Cancer Research and Clinical Oncology, 
139, 419-427. https://doi.org/10.1007/s00432-012-1345-5

[14] Findlay, M., Purvis, M., Venman, R., Luong, R. and Carey, S. (2020) Nutritional Management of Patients with Oesophageal Cancer throughout the Treatment Trajectory: Benchmarking against Best Practice. Supportive Care in Cancer, 28, 5963-5971. https://doi.org/10.1007/s00520-020-05416-x

[15] Bargetzi, L., Brack, C., Herrmann, J., Bargetzi, A., Hersberger, L., Bargetzi, M., et al. (2021) Nutritional Support during the Hospital Stay Reduces Mortality in Patients with Different Types of Cancers: Secondary Analysis of a Prospective Randomized Trial. Annals of Oncology, 32, 1025-1033.

https://doi.org/10.1016/j.annonc.2021.05.793

[16] Castillo-Martínez, L., Castro-Eguiluz, D., Copca-Mendoza, E.T., Pérez-Camargo, D.A., Reyes-Torres, C.A., Ávila, E.A.-D., et al. (2018) Nutritional Assessment Tools for the Identification of Malnutrition and Nutritional Risk Associated with Cancer Treatment. Revista de Investigación Clínica, 70, 121-125.

https://doi.org/10.24875/RIC.18002524

[17] Bauer, J., Capra, S. and Ferguson, M. (2002) Use of the Scored Patient-Generated Subjective Global Assessment (PG-SGA) as a Nutrition Assessment Tool in Patients with Cancer. European Journal of Clinical Nutrition, 56, 779-785.

https://doi.org/10.1038/sj.ejcn.1601412

[18] Zhang, L., Lu, Y. and Fang, Y. (2014) Nutritional Status and Related Factors of Patients with Advanced Gastrointestinal Cancer. British Journal of Nutrition, 111, 1239-1244. https://doi.org/10.1017/S000711451300367X 